\title{
THE RADIOPACITY OF DENTAL PROSTHESES (FIXED AND REMOVABLE) ON PLAIN RADIOGRAPHS - AN EXPERIMENTAL STUDY
}

NH Abu Kasim, BJJ Abdullah, J Mahadevan \& N Yunus. The Radiopacity of Dental Prostheses (Fixed and Removable) on Plain Radiographs - An Experimental Study, Annals Dent Univ Malaya 1998; 5: 35-39

\begin{abstract}
The impaction of dental prostheses in either the airway or esophagus is an under-recognized problem which may result in severe morbidity or even mortality. The radio-opacity and the size of fixed and removable dental prostheses in an animal carcass was investigated. Prostheses were placed one at a time in the oro-laryngopharynx or in tho trachea and the esophagus. Lateral radiographs were taken for each prosthesis in site. The radio-opacity and size of the prostheses on the radiograph was graded. Most of the prostheses investigated were radio-opaque though the removable prostheses were more likely to be radiolucent and differ in size. In a symptomatic patient with a missing dental prosthesis, a negative chest or abdominal radiograph does not exclude impaction, inhalation or ingestion. Further evaluation with endoscopy or even computed tomography may be essential to reduce the possibility of severe morbidity or even mortality.
\end{abstract}

Keywords: Radiology, radiopacity, dentat prostheses, in vitro

\section{INTRODUCTION}

Foreign body $(\mathrm{FB})$ ingestion with impaction in either the esophagus or bronchial tree is a relatively common problem presenting to the Accident and Emergency departments. The most common causes are usually due to either fish or chicken bones in adults while in children these tend to be coins, button batteries, toys etc.. The impaction of dental prosthesis is relatively uncommon but not rare though it is under-recognized or under-reported. Review of the literature $(1,2)$ showed that the type of dental prosthesis most commonly impacted is the partial or broken denture. There has however been a reported case of a full denture impacted in the trachea in a patient following a stroke which was not diagnosed till late (3). The soft tissue lateral radiograph in addition to the chest and abdominal radiographs is routinely done to assess patients with ingested or impacted foreign body. Though the role of plain film in the diagnosis and management of impacted foreign bodies is questioned, it must be admitted that the definite presence of an impacted FB would allow a more definitive plan of management. In addition it may detect any complications of the impaction like subcutaneous emphysema, pneumothorax, lung abscesses, segmental or lobar atelectasis or even free intraperitoneal air. However in the absence of radiographic evidence of impacted FB an endoscopic examination must be carried out to reduce the likelihood of severe morbidity or even death. There is however no study done to assess the radio-opacity, size and shape of dental prostheses on plain radiographs.

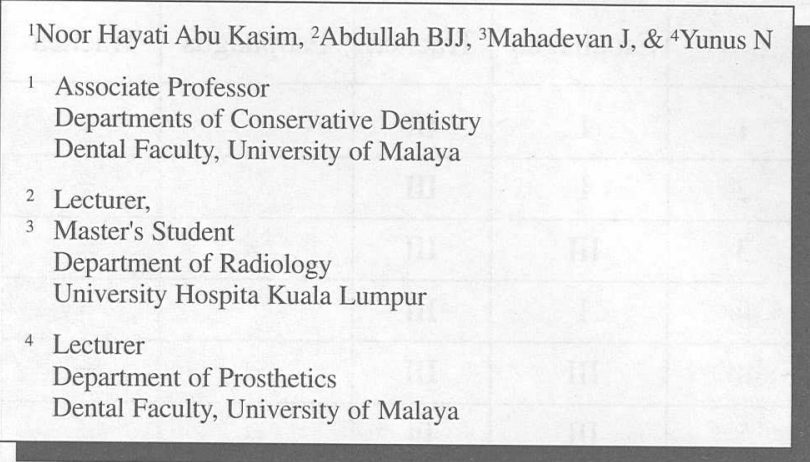

The purpose of the study was to determine the radioopacity and radiographic size of different types of dental prostheses (fixed and removable) when placed in an animal carcass.

\section{MATERIALS AND METHOD}

A total of 14 different types of fixed and removable dental prostheses were taken and divided into two groups, Group A and B respectively (Tables I \& II). The fixed prostheses (Group A) included a full porcelain crown, a porcelain jacket crown, a porcelain fused to metal crown, a temporary bridge, a fixed-fixed bridge with implant attachment, a resin retained hybrid anterior bridge and a fixed-fixed anterior bridge. The removable prostheses (Group B) consisted of an

Table I. List of the fixed dental prostheses (Group A)

\begin{tabular}{|c|l|}
\hline 1 & Full gold crown \\
\hline 2 & Porcelain jacket crown \\
\hline 3 & Porcelain fused to metal; crown \\
\hline $4 \mathrm{a}$ & Ternporary bridge \\
\hline $4 \mathrm{~b}$ & Fixed-fixed bridge with implant attachmen \\
\hline 5 & Resin-retained hybrid anterior bridge \\
\hline 6 & Fixed-fixed anterior bridge \\
\hline
\end{tabular}

Table II. List of the removable dental prostheses (Group B)

\begin{tabular}{|l|l|}
\hline 1 & Lower partial acrylic denture \\
\hline 2 & Upper sectional denture - Dame \\
\hline 3 & Upper sectiona1 denture - tooth \\
\hline 4 & Removable orthodontic appliance \\
\hline 5 & Lower chrome partial denture \\
\hline 6 & Full acrylic denture \\
\hline 7 & Upper partial acrylic denture \\
\hline
\end{tabular}


Table III. Radio-opacity and size of fixed (Group A) prostheses in the trachea and esophagus.

\begin{tabular}{|c|c|c|c|c|}
\hline \multirow{2}{*}{ Number } & \multicolumn{2}{|c|}{ Radio-opacity } & \multicolumn{2}{c|}{ Size } \\
\hline & Esophagus & Trachea & Esophagus & Trachea \\
\hline 1 & I & III & & $=$ \\
\hline 2 & I & III & & $=$ \\
\hline 3 & III & III & $=$ & $=$ \\
\hline $4 \mathrm{a}$ & I & III & & $=$ \\
\hline $4 \mathrm{~b}$ & III & III & $=$ & $=$ \\
\hline 5 & III & III & $=$ & $=$ \\
\hline 6 & III & III & $=$ & $=$ \\
\hline
\end{tabular}

lower partial acyrlic denture, an upper sectional denture (frame and tooth), removable orthodontic appliance, a chrome partial denture, a full acrylic denture and an upper acrylic partial denture.

Each of the prostheses from group A was placed in turn in the trachea and esophagus of an animal carcass and radiographs exposed. The carcass consisted of the head and entire neck of a local goat which had been sacrificed. The carcass was sectioned using the mid sagittal plane through the pharynx, larynx and esophagus to allow placement of the dental prostheses. The goat carcass was placed in formalin to pre- serve it and prevent excessive drying. The radiographic factors used for the entire study was determined by exposing the carcass using different radiographic factors to find those which were the best at demonstrating the soft tissue of the neck. The $\mathrm{kVp}$ was 40 while $4.5 \mathrm{mAs}$ was used. The film focus distance was fixed at $100 \mathrm{~cm}$. No grids were used. The film use was Kodak X-Omatic while the intensifying screen was Kodak X-Omatic Regular. Since the Group B prostheses were larger they could only be placed in the oro-laryugopharynx, otherwise the two halves of the carcass could not be opposed.

The radio-opacity of the prostheses was graded as Grade I being not visible, Grade II as being moderately visible and Grade III being clearly visible. In addition the size of each of the prosthesis was also graded; = indicating no difference in size while a $\downarrow$ indicated a smaller size on the radiographs.

\section{RESULTS}

Almost all the fixed prostheses (Group A) were clearly visible in the trachea and esophagus except for three [Full gold crown (Figure 1), porcelain jacket crown and the temporary bridge (Figure 2)] which were not visible in the esophagus (Table III). There was however no difference in the size of those that were visible. For the removable prostheses (Group B) placed in the oro-laryngopharynx, the full acrylic denture was not visible while the upper partial acrylic denture (Figure 3 ) was moderately visible (Table IV). The rest of the removable prostheses were clearly visible. As regards the differences in size, only one (upper sectional denture frame) was

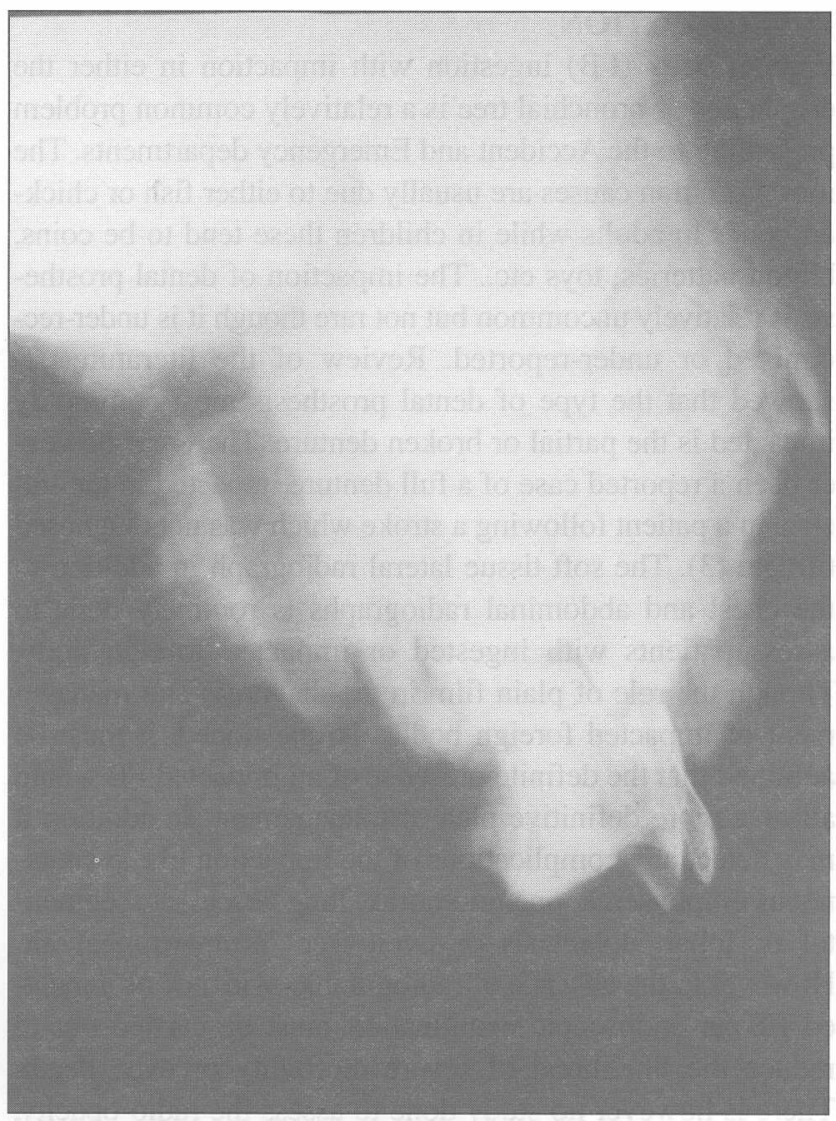

Figure $1 a \& b$. The full gold crown (black arrow) is clearly in the trachea but not visualized in the oesophagus. 

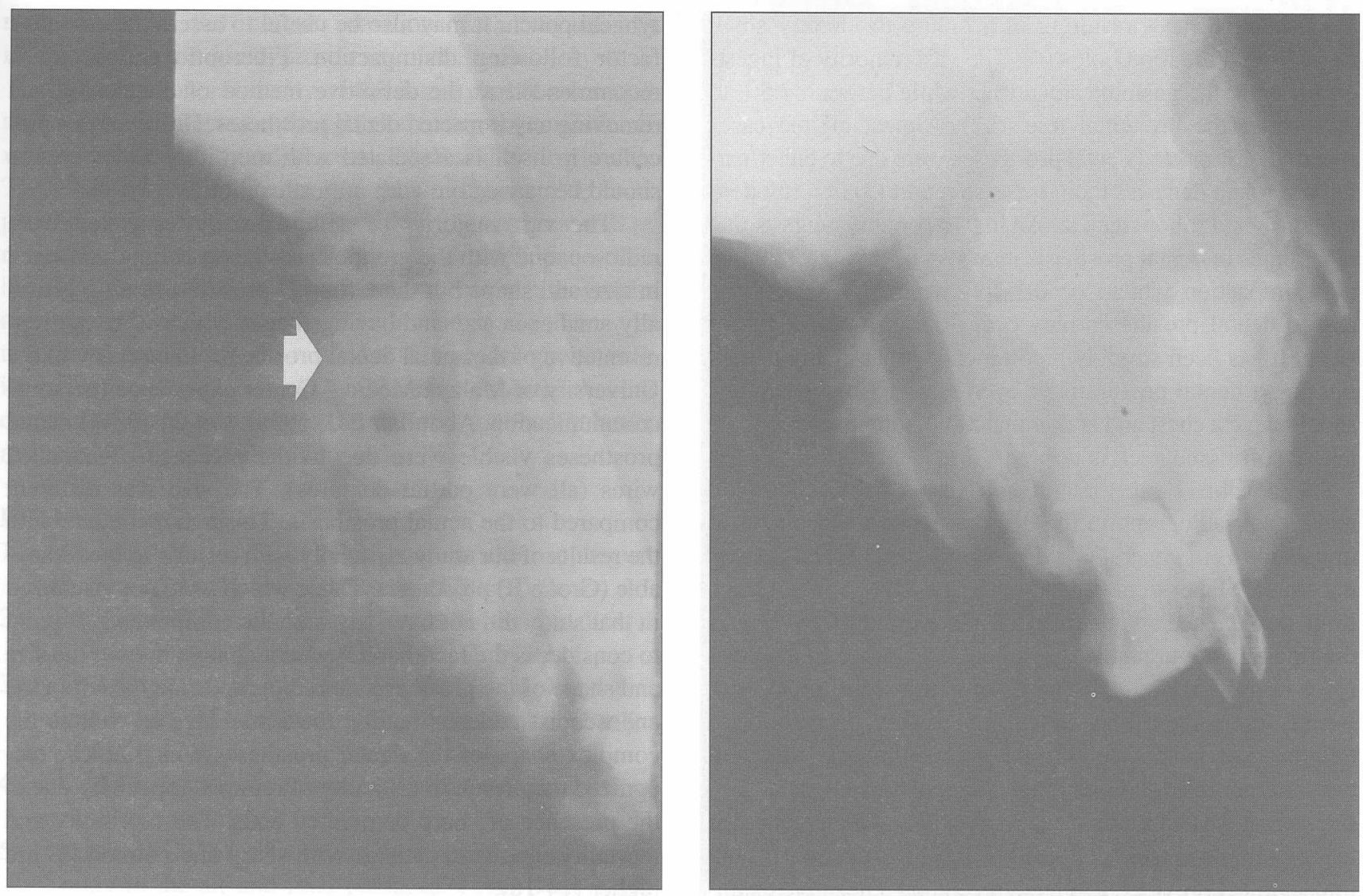

Figure $2 a \& b$. The temporary bridge (white arrow) seen in the trachea but again not visible when placed in the esophagus

Table IV. Radio-opacity and size of removable (Group B) prostheses placed in the oro-laryngopharynx.

\begin{tabular}{|c|c|c|}
\hline Number & Radio-opacity & Size \\
\hline 1 & II & $\downarrow$ \\
\hline 2 & III & $=$ \\
\hline 3 & III & $\downarrow$ \\
\hline 4 & III & $\downarrow$ \\
\hline 5 & III & $\downarrow$ \\
\hline 6 & I & $\downarrow$ \\
\hline 7 & III & \\
\hline
\end{tabular}

of the same size while the rest were smaller in size as only the metal component was seen.

\section{DISCUSSION}

Even though the impaction of FB's in the esophagus or trachea is recognized as being a common problem presenting to the Accident \& Emergency department, those due to dental prostheses is not highlighted. The incidence varies from $0.4 \%$ (1) to $13 \%$ (personnel communication Abdullah BJJ, 1997). Of all the factors associated with FB ingestion wearing a denture is one of the commonest associations $(4,5)$. This has been attributed to loss of the normal tactile sensations of

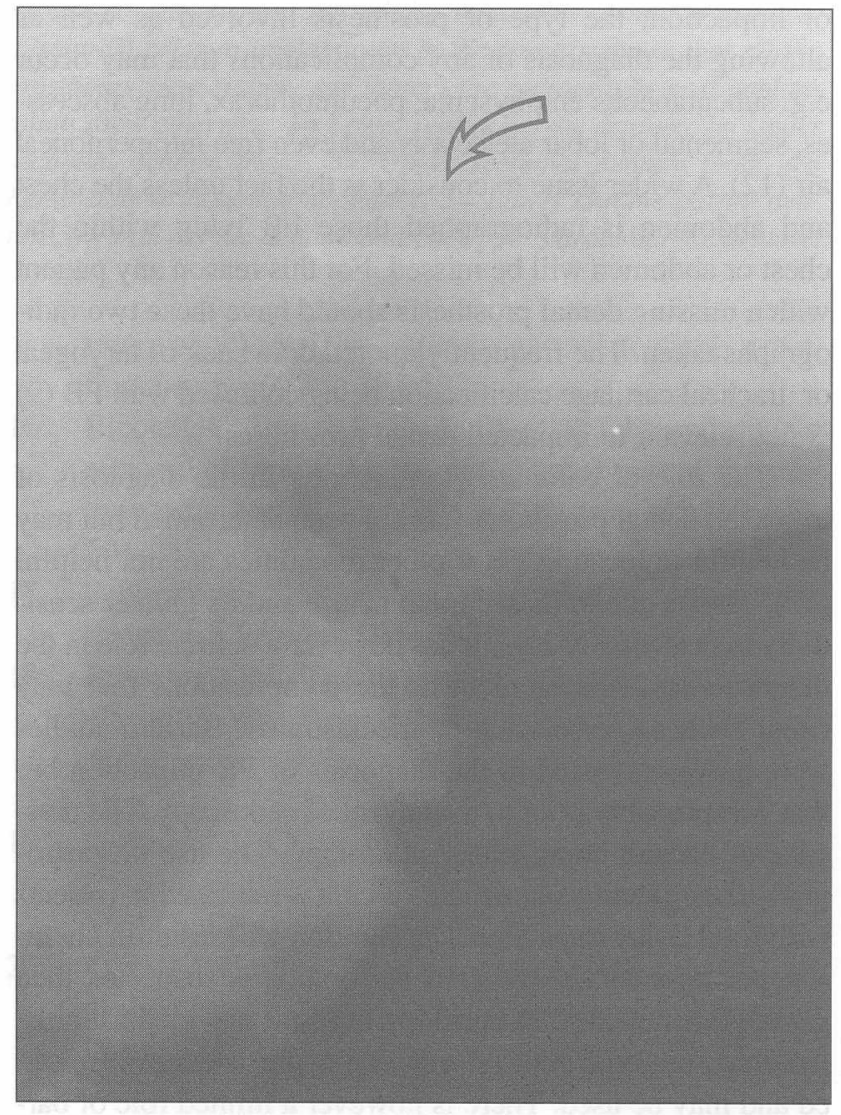

Figure 3. Upper partial acrylic denture (open black arrow) visualized due to the presence of metal clasps 
the palatal surfaces resulting in a failure to identify small items within the food bolus (6). The vast majority of ingested FB enter the gastointestinal tract while between $7.5 \%$ to $20 \%$ enter the bronchial tree $(6,7)$. Almost all the cases reported of impacted dental prostheses were due to either partial or broken dentures though a case report (3) of a full denture impacted following a stroke in two patients suggests that other types of dental prosthesis may also be involved. Unlike bone impaction which: occurs following ingestion, those due to dental prostheses may occur during trauma, fits or sleep. It has been strongly recommended that in any patient missing a dental prosthesis or even teeth following trauma should have a chest and abdominal radiograph to check if the lateral soft tissue neck is normal.

The value of the lateral soft tissue neck, chest and abdominal radiograph in the diagnosis and management of impacted FB in general and dental prostheses specifically is uncertain. Marais et al $(8)$ and others $(9,10)$ feel that it has a limited role since a large proportion are radioluscent as was the findings in our study. It resulted in a change in management in only $1.4 \%$ and unnecessary endoscopy in $2 \%$ (9). Further more there may be an unnecessary delay prior to treatment being carried out. The common radiographic features are not reliable in making a diagnosis of impacted FB. In contrast we agree with McGill et al (11) who feel that the lateral soft tissue neck is basic to the investigation of any impacted FB provided it is recognized that a negative examination does not exclude a FB. A definitive radiographic diagnosis of impacted dental prostheses would indicate the level of impaction, the type of prosthesis involved as well as allowing the diagnosis of any complications that may occur e.g. subcutaneous emphysema, pneumothorax, lung abscesses, segmental or lobar atelectasis and even free intraperitoneal air (12). A wider issue to consider is the fact unless the chest and abdomen is radiographed those FB lying within the chest or abdomen will be missed. For this reason any patient with a missing dental prosthesis should have these two radiographs taken. The frequently quoted drawback of laryngeal or tracheal cartilage calcification being confused with FB (9) is not relevant to impacted dental prostheses.

The role of computed tomography in the diagnosis of impacted dental prostheses has not been determined but may be helpful in locating FB if other modalities are not helpful (13). This is due to its sectional nature and its greater sensitivity in detecting X-rays. It has however a definite role in the diagnosis and management of the complications that may occur such as perforation or mediastinitis. Barium studies were previously used in the diagnosis of $\mathrm{FB}$ impaction but that was probably prior to the advent of endoscopy. The presence of barium complicates endoscopy. The use of gastrograffin (meglumine diatrizoate) is contra-indicated in patients with food bolus impaction as aspiration will result in chemical pneumonitis. However if perforation is suspected then gastrograffin is ideal as barium will result in a mediastinitis. Isotonic non-ionic contrast medium is much safer if aspirated and may be used. There is however a limited role of barium swallows in determining the level of impaction and to forewarn the clinician about the potential hazard of a pha- ryngeal pouch. It may also be useful to assess the causative factor following disimpaction. Fiberoptic endoscopy is recommended as the definitive method of diagnosing and removing any impacted dental prostheses. However this procedure in itself is associated with morbidity and therefore should be carried out with utmost caution.

The vast majority of dental prostheses tested were radio-opaque with the group A prostheses being unchanged in size and shape but the Group B prostheses being generally smaller in size and having shapes which were not representative of the actual dental prosthesis. In a review of the University of Malaya Medical Center experience (personal communication, Abdullah BJJ, 1997) 7 of 21 (33\%) dental prostheses visible were due to the presence of attached wires (all were partial dentures). The size was different compared to the actual prosthesis. This again concurs with the results of our study especially with regards to the removable (Group B) prostheses. Those which were not visualized in that study did not have any metallic components. A point to consider is the fact that a frequent change in both the size and shape of the prosthesis on radiographs may result in the endoscopist underestimating the actual size as well as the complex shape of the dental prostheses which unless recognized may result in difficulty of removal especially due to the presence of sharp or pointed ends. The morbidity and mortality figures in patients with sharp and pointed FB are higher (14-16)

There is an important need for all dental prostheses to have radio-opaque material inserted into the dental prostheses at the time of fabrication especially if it is completely radioluscent to reduce the likelihood of being missed $(17,18)$. Heavy metal salts as well as glass fillers have been added to the base material resin to achieve opacity on radiographs. The most promising material is silanated, barium fluoride impregnated powdered glass to clear poly methyl methacrylate denture resin material. This would not increase the cost but may prove very useful.

A common misconception is that if a dental prosthesis is visible on intra-oral or occlusal films than it should be visible on standard radiographs which has been proved in this study (Figure 4). The visibility of an object depends on the differences in density as well as the differences in thickness. Thus two objects with differing densities may not be radiographically visible if the differences in thickness compensate. This was seen in the present study where the full gold crown, porcelain jacket crown and the fixed-fixed bridge with implant attachment were clearly seen in the trachea but not visible in the esophagus.

Several of the studies evaluating radiopacity of denture liner have only used occlusal films with the prosthesis placed in the oral cavity $(19,20)$. We have used an animal carcass to simulate as closely as possible the human situation. The anatomy of the oro-laryngopharyx was not distorted. The exposure factors used were also similar to that used for humans. Previous studies to assess radiopacity of fish bones have used the reconstituted offal of pigs and even the thighs of chicken (10) which are not truly representative. We therefore feel that the phantom used in this study is closely rep- 
resentative of the actual situation with regards to ingested/impacted dental prostheses in the neck.

In conclusion a majority of the dental prostheses evaluated were radio-opaque though the removable prosthesis were more likely to be radiolucent as well as differing in size. The visibility was due to the presence of the metallic components. This reemphasizes the need for the base material of removable prostheses to be radiopaque. In a patient with a history of impacted dental prostheses where the radiographs are negative, an endoscopic examination is mandatory to reduce the possibility of a missed or delayed diagnosis with its antecedent complications. It is therefore essential that the dental profession encourages the industry to develop better methods of fragment identification.

\section{REFERENCES}

1. Nandi P, Ong GB. Foreign body in the esophagus: review of 2394 cases. Br J Surg 1978;6S:5-9.

2. Phillipps JT, Patel P. Swallowed foreign bodies. J Laryngology and Otology 1988;102:235-241.

3. Fort S, Batty GM, WiLkins WE. Prolonged pharyngeal impaction of dentures following stroke. Stroke 1989;20:1748-1750.

4. Gunn A. Intestinal perforation due to swallowed fish or meat Lancet 1966;1: 125-8.

5. Bunker PG. The role of dentistry in problems of foreign body in the air and food passages. J Am Dent Assoc 1962;64:782-7.

6. Koch H. Operative endoscopy Gastrointest Endosc 1977;24:65-8.

7. Webb WA. Management of foreign bodies in the upper gastrointestinal tract: Update. Gastrointest Endosc 1995;41:39-51.

8. Marais J, Mitchell R, Wightman AJA. The value of radiographic assessment of oropharyngeal foreign bodies. J of Laryngology \& Otology 1995;109:452-454.

9. Jones NS, Lanningan FJ, Salaama NY. Foreign bodies in the throat: a prospective study of 388 cases. J Laryngology \& Otology 1991;105:104-108.

10. E11 SR. Radiopacity of fish bones. J Laryngology and Otology 1989;103:1224-1226.

11. McGill TJ, Ohlms L. Foreign bodies in the upper aerodigestive tract. In Otolaryngology-Head and Neck Surgery. 2nd. Edition (Cummings CW, Frederickson JM, Marker LA et al eds) Mosby Year Book St. Louis 3:2396-2400.

12. Taylor RB. Esophageal foreign bodies. Emergency Medicine Clinics of North America 1987;5:301-311.

13. Newton JP, Abel CH, Yemm R. The use of computed tomography in the detection of radiolucent denture base material in the chest. J Oral Rehab 1987;14:193202.

14. Carp L. Foreign bodies in the intestine. Ann Surg 1927;85:575-91.

15. Rosch W, Classen M. Fiberendoscopic foreign body

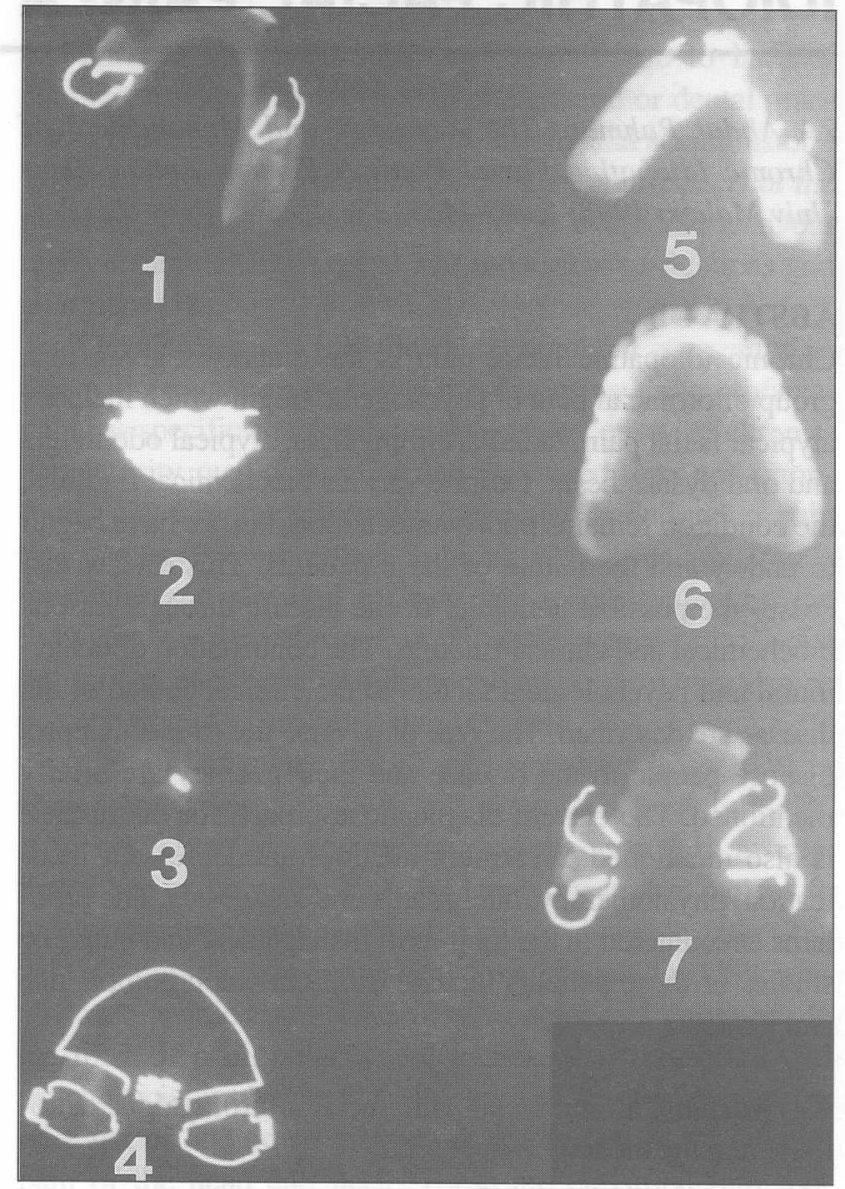

Figure 4. The radiograph of the removable dental prostheses (Group B) prior to implantation in the carcass shows that all the prosthoses were radiopaque though the radiographs post implantation show otherwise. $1=$ Lower partial acrylic denture, $2=$ Upper sectional denture frame, $3=$ Upper sectional denture tooth, $4=$ Removable orthodontic appliance, $5=$ Lower chrome partial denture, $6=$ Full acrylic denture and $7=$ Upper partial acrylic denture.

removal from the upper gastrointestinal tract. Endoscopy 1972;4: 193-197.

16. Ricote GC, Torre LR, DeAyala et al. Fiberendoscopic removal of foreign bodies of the upper part of the gastrointestinal tract. Surg Gynecol Obstet 1985;160:499504.

17. Schneide SS, Roistacher S. Aspiration of denture base material. J Prosthet Dent 1971:25;493.

18. Braucher GM. The desirability of using radiopaque plastics in dentistry - A status report. The Council on dental materials, instruments, and equipment. J Am Dent Assoc 1981:102;347.

19. Goshima T, Gettleman, Goshima Y, Yamamoto A. Evaluation of radiopaque denture liner. Oral Surg Oral Med Oral Pathol 1992;74:379-82.

20. Tsao DH, Guilford HJ, Kazanoglu A, Bell DH. Clinical evaluation of radiopaque denture base resin. J Prosthet Dent 1984;51:456-458. 